\title{
Exact mechanical models of fractional hereditary materials
}

\author{
Mario Di Paola ${ }^{a)}$ \\ Dipartimento di Ingegneria Civile, Ambientale ed Aerospaziale, dei Materiali \\ (DICAM), Viale delle Scienze Ed.8, I-90128 Palermo, Italy \\ Massimiliano Zingales ${ }^{\mathrm{b})}$ \\ Dipartimento di Ingegneria Civile, Ambientale ed Aerospaziale, dei Materiali \\ (DICAM), Viale delle Scienze Ed.8, I-90128 Palermo, Italy and Istituto \\ Euromediterraneo di Scienza e Tecnologia (IEMEST), Via E. Amari 123, \\ I- 90139 Palermo, Italy
}

(Received 22 September 2011; final revision received 2 April 2012;

published 19 June 2012)

\begin{abstract}
Synopsis
Fractional Viscoelasticity is referred to materials, whose constitutive law involves fractional derivatives of order $\beta \in \mathbb{R}$ such that $0 \leq \beta \leq 1$. In this paper, two mechanical models with stressstrain relation exactly restituting fractional operators, respectively, in ranges $0 \leq \beta \leq 1 / 2$ and $1 / 2 \leq \beta \leq 1$ are presented. It is shown that, in the former case, the mechanical model is described by an ideal indefinite massless viscous fluid resting on a bed of independent springs (Winkler model), while, in the latter case it is a shear-type indefinite cantilever resting on a bed of independent viscous dashpots. The law of variation of all mechanical characteristics is of power-law type, strictly related to the order of the fractional derivative. Because the critical value $1 / 2$ separates two different behaviors with different mechanical models, we propose to distinguish such different behavior as elasto-viscous case with $0 \leq \beta \leq 1 / 2$ and visco-elastic case for $1 / 2 \leq \beta \leq 1$. The motivations for this different definitions as well as the comparison with other existing mechanical models available in the literature are presented in the paper. (C) 2012 The Society of Rheology. [http://dx.doi.org/10.1122/1.4717492]
\end{abstract}

\section{INTRODUCTION}

Stress decay under constant strain (relaxation) as well as strain increments under constant stress (creep), observed in real materials, has fascinated several leading scientists from the late 19th century aiming toward the mechanical characterization of timedependent material behavior. Relaxation and creep function of materials have been modeled in scientific literature, mainly, by means of single and/or linear combination of exponential functions in the attempt to capture both solid and fluid phases. Such an

\footnotetext{
a)Electronic mail: mario.dipaola@unipa.it

b) Author to whom correspondence should be addressed; electronic addresses: massimiliano.zingales@unipa.it and massimiliano.zingales@iemest.edu
} 
approach does not allow for a correct fit of experimental results and in the last century it has been observed that stress relaxation and creep function of real-type materials decays with power-law [see Nutting (1921)]. Later on it has been shown that the transfer function of VE materials may be identified in frequency domain by means of real powers of frequency leading, by inverse Fourier transform, to fractional operators in time domain [Scott-Blair (1949)] with real-order $\beta$ : $0 \leq \beta \leq 1$. Since then a lot of researches on this subject have been carried out enforcing the knowledge of hereditary materials in time domain [Blizard (1951); Rouse and Sittel (1953); Caputo (1974); Bagley and Torvik (1983, 1986)] and in frequency domain [Spanos and Evangelatos (1991)]. The limit cases $\beta=0$ and $\beta=1$ correspond to pure elastic solid and pure viscous fluid, respectively. This consideration leads to conclude that the hereditary material behavior is intermediate between these limit cases and therefore the most appropriate rheologic representation must involve fractional operators. Similar considerations about fractional-order operators may be framed in the context of renewal processes of continuous time random walk (CTRW) as it has been observed in Metzler and Klafter (2000).

Rheologic relations involving fractional operators do not possess, to the best of authors' knowledge, a correspondent mechanical model and a symbolic element, dubbed springpot [after Scott-Blair (1949)] has been introduced at the mid of the last century. The springpot may also be combined with other, conventional, mechanical elements as dashpots and/or linear springs to model nonvanishing long-term relaxation [Metzler et al. (1995a, 1995b); Metzler and Nonnenmacher (2003)]. A mechanical model capable to represent the springpot fractional elements in terms of a proper arrangement of springs and dashpots has not been reported in scientific literature unless for derivative of orders 3/2. In this latter case, it has been proved [see Bagley and Torvik (1983)] that the shear stress applied on a massless plate resting on an half-space filled by Newtonian fluid with prescribed transverse motion is ruled by a fractional derivative of order $1 / 2$ of the strain rate (order $3 / 2$ of the strain).

Several attempts to yield fractional-order models with exponents different by $3 / 2$ have been proposed in the past, from the beginning of the 1990s [Glockle and Nonnemacher (1991); Schiessel and Blumen (1993, 1994) introducing fractance-type models composed by springs and dashpots that are repetitions of a single unit [Friedrich (1991), (1993); Friedrich and Braun (1994); Metzler et al. (1995); Heymans and Bauwens (1994); Sakakibara (2001); Podlubny (1998); Heymans (2003)]. At the limit, by increasing the number of such units, at steady-state, the constitutive equation for such a fractance model reverts to an approximation of fractional operator.

The present study aims to introduce an exact mechanical model of fractional viscoelastic (VE) material. This result has been achieved in three steps:

- In the first step, the Bagley-Torvik (BT) model has been properly modified to yield an exact mechanical model of hereditariness with a rheologic relation involving fractional derivatives of order $\beta=1 / 2$. This model is obtained by means of an unbounded, shear-type, massless elastic column resting on a bed of independent linear dashpots.

- In the second step, we observe that the Schiessel-Blumen (SB) fractal ladder model [Schiessel and Blumen (1994); Metzler et al. (1995a, 1995b)] is completely coalescing with a discrete shear-type linear elastic model externally restrained by linear dashpots, that is, in turn, completely equivalent to the discretized version of the modified BT model. The only difference with BT model is that springs and dashpot coefficients in the SB model are nonuniform along the ladder. The main drawback involved in SB model is that the fractal ladder yields only an approximation of fractional derivatives at steady-state. 
- As soon as we realized that a suitable modification of stiffness and damping coefficients of the discretized version of the BT model, as in the SB model, corresponds to approximations of fractional-order operators, in the third step, we look for the functional class of stiffness and viscosity of the model to yield, exactly, fractional-order operators.

In this regard, we propose two mechanical models: (i) If the differentiation order $\beta$ is $\beta \in[0,1 / 2]$ then the mechanical model is composed by a column of massless Newtonian fluid resting on a bed of independent linear springs and (ii) If $\beta \in[1 / 2,1]$, then the correspondent mechanical model is a massless, shear-type column resting on a bed of linearly independent dashpots. By assuming that all the mechanical characteristics of the two models decay with power-law as the distance from the upper boundary increases, then a Caputo' fractional derivative relating the stress at the top of the model to the corresponding strain is exactly obtained. It is shown that, if the exponent of the power-law tends to zero (that is all the mechanical characteristics are uniform) then a fractional derivative of order 1/2 appears as in the modified BT model.

As the viscosity between shear layers disappears, then the pure solid constitutive relation is recovered $(\beta=0)$. If, instead, the shear modulus of elasticity in the cantilever model disappears, then the pure fluid behavior is recovered. Because the critical value $\beta=1 / 2$ separates two different behaviors with different mechanical models, we propose to denote elasto-viscous (EV) materials as $\beta \in[0,1 / 2]$ and VE materials as $\beta \in[1 / 2,1]$. This is due to the fact that in the EV materials the elastic component prevails over the viscous characteristics while for VE materials the viscous feature prevails over the elastic ones as shown in Sec. V.

\section{THE FRACTIONAL MODEL OF HEREDITARINESS}

It is widely understood that stress-strain relations of hereditary materials involve fractional operators. The simplest way to show this behavior is provided by the observation of creep or relaxation tests performed on hereditary specimens. At the beginning of the 20th century, Nutting observed that the relaxation function $G(t)$ obtained from experiments on polymeric materials is well fitted by a power law, that is,

$$
G(t)=\frac{C_{\beta}}{\Gamma(1-\beta)} t^{-\beta}
$$

where $\Gamma(\cdot)$ is the Euler-Gamma function (see Appendix A), $C_{\beta} / \Gamma(1-\beta)$ and $\beta$ are characteristic coefficients depending on the material at hand that must satisfy the thermodynamic restrictions $\beta \in[0,1]$ and $C_{\beta}>0$. Once the relaxation test is performed, the creep function of the material, dubbed $J(t)$, may be predicted since in Laplace domain the following relationship:

$$
\hat{G}(s) \hat{J}(s)=\frac{1}{s^{2}}
$$

holds true, where we denoted $s$ is the Laplace parameter, $\hat{G}(s)$ and $\hat{J}(s)$, are the Laplace transform of the relaxation and the creep function, respectively, (see Appendix A).

The Laplace transform of the relaxation function $G(t)$ expressed in Eq. (2.1) is represented as 


$$
\hat{G}(s)=C_{\beta} s^{\beta-1}
$$

then, in virtue of Eq. (2.2), $\hat{J}(s)$ is provided in the form

$$
\hat{J}(s)=\frac{1}{C_{\beta} s^{\beta+1}},
$$

whose inverse Laplace transform yields the creep function $J(t)$ as

$$
J(t)=\frac{1}{C_{\beta} \Gamma(1+\beta)} t^{\beta} .
$$

Thermodynamic restrictions of the range of possible values of the decaying exponent $\beta$ as well as on values of the force coefficient $C_{\beta}$ do not allow for any mechanical consideration about fractional-order VE materials and henceforth symbols reported in previous scientific literature are consistent with Eqs. (2.1) and (2.5).

With this machinery in mind, due to the Boltzmann superposition principle for a system with $\varepsilon(0)=0$ the stress $\sigma(t)$ and the strain $\gamma(t)$ are related each other by the following convolution integral:

$$
\sigma(t)=\int_{0}^{t} G(t-\bar{t}) \dot{\gamma}(\bar{t}) d \bar{t}
$$

where $\gamma(t)$ is the assigned strain history. By inserting Eq. (2.1) into Eq. (2.6), we obtain

$$
\sigma(t)=\frac{C_{\beta}}{\Gamma(1-\beta)} \int_{0}^{t} \frac{\dot{\gamma}(\bar{t})}{(t-\bar{t})^{\beta}} d \bar{t} .
$$

A close observation of Eq. (2.7) reveals that the convolution integral coalesces with the Caputos' fractional derivative labeled as $\left({ }_{C} D_{0^{+}}^{\beta} \gamma\right)_{t}(t)$ (Appendix A for details) and then, the constitutive law is provided in the form

$$
\sigma(t)=C_{\beta}\left({ }_{C} D_{0^{+}}^{\beta} \gamma\right)(t)
$$

from Eq. (2.8) we may assert that for a material whose relaxation function is well suited by Eq. (2.1) in power-laws functional class, then the constitutive law in terms of Caputo's fractional derivatives is readily obtained.

On the other hand, using again the Boltzmann superposition principle, for the case in which the stress history is assigned and the corresponding strain history has to be evaluated, we may write

$$
\gamma(t)=\int_{0}^{t} J(t-\bar{t}) \dot{\sigma}(\bar{t}) d \bar{t}
$$

The stress-strain law represented in Eq. (2.9) is valid for $\sigma(0)=0$. By inserting Eq. (2.5) into Eq. (2.9), we obtain

$$
\gamma(t)=\frac{1}{C_{\beta} \Gamma(\beta+1)} \int_{0}^{t}(t-\bar{t})^{\beta} \dot{\sigma}(\bar{t}) d \bar{t}=\frac{1}{C_{\beta} \Gamma(\beta)} \int_{0}^{t}(t-\bar{t})^{\beta-1} \sigma(\bar{t}) d \bar{t},
$$


so that, recalling the definition of the Riemann-Liouville fractional integral $\left(I_{0^{+}}^{\beta} \sigma\right)(t)$ reported in the Appendix [see, e.g., Samko et al. (1993) for details], the constitutive law is written as

$$
\gamma(t)=\frac{1}{C_{\beta}}\left(I_{0^{+}}^{\beta} \sigma\right)(t) .
$$

Previous formulations in terms of Caputo' fractional derivatives hold true also in presence of nonvanishing initial conditions, namely, $\gamma(0)=\gamma_{0} \neq 0$ and then in Eq. (2.8) an additional term that reads $G(t) \gamma_{0}=\gamma_{0} t^{-\alpha} / \Gamma(1-\alpha)$ has to be added; If, instead, $\sigma(0)=\sigma_{0} \neq 0$ then the additional term $J(t) \sigma_{0}$ has to be added in Eq. (2.11). An equivalent formulation of the fractional model of hereditariness may be obtained in term of the more familiar Riemann-Liouville fractional derivatives $\left(D_{0^{+}}^{\beta} \gamma\right)(t)$ accounting for the initial condition $\gamma(0)=\gamma_{0} \neq 0$ by means of the relation

$$
\left({ }_{C} D_{0^{+}}^{\beta} \gamma\right)(t)=\left(D_{0^{+}}^{\beta} \gamma\right)(t)-\frac{\gamma_{0}}{\Gamma(1-\alpha) t^{\alpha}} .
$$

Summing up: By assuming that the creep and relaxation functions belong to a power-law functional class, then the rheologic model involves Caputo' fractional operators. It is to be emphasized that the Caputo' fractional derivatives coalesce with the RiemannLiouville fractional derivative only for a quiescent system at $t=0$ or for systems operating from $t=-\infty$; In other cases, a relation involving Riemann-Liouville operators may be formulated by means of Eq. (2.12).

Values of coefficient $\beta$ coinciding with the extrema of the closed interval $[0,1]$ correspond to asymptotic behavior of the rheologic VE model expressed by power-law assumption that is: pure elastic solid for $\beta=0$ and pure viscous fluid for $\beta=1$. Values of $\beta \in[0,1]$ correspond to an intermediate behavior between purely elastic solid and purely viscous fluid as for complex-structured materials as well as for soft matter and it may be experienced, also, in damping of elastic waves propagation [Mainardi (2010)] and in the relaxation time spectrum of filled rubbers [Metzler et al. (1995b)].

The use of fractional rheologic model discussed in this section may also be combined with other nonfractional elements to represent more sophisticated material behavior. Indeed, fractional elements may be placed in parallel/series with a spring so ensuring a nonvanishing value of the relaxation modulus or a finite value of the instantaneous modulus [Metzler et al. (1995a, 1995b)]. Combination of fractional and nonfractional elements leads to relaxation/creep function provided by analytical expressions involving MittagLeffler function that represents the Green' function of a two-or-more terms linear fractional differential equation [Podlubny (1998); Mainardi (2010)].

\section{REVISITATION OF MECHANICAL FRACTIONAL MODELS}

This section is devoted to the analysis of two existent mechanical models of fractional hereditary materials so that a proper path to the proposed exact model of fractional hereditariness may be introduced in Sec. IV. The BT and the SB models are discussed, respectively, in Secs. III A and III B.

\section{A. The BT model revisited and modified}

The BT model is represented by a thin massless plate resting on a perfectly Newtonian viscous half-space (see Fig. 1). Let $\mathrm{z}$ be the vertical axis as shown in Fig. 1, $u(z, t)$ 


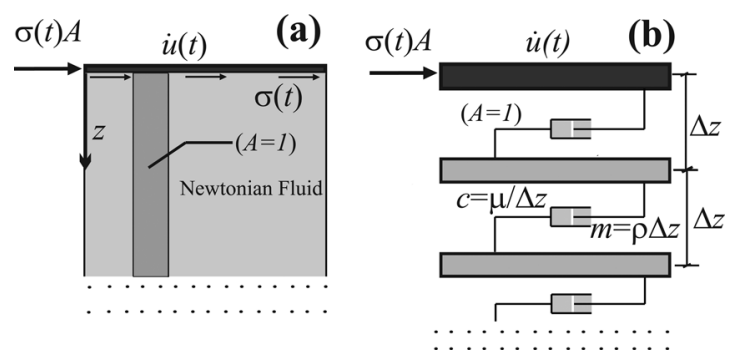

FIG. 1. ( a) Original BT model; (b) discretized version of the BT model.

denotes the transverse displacement of fluid layers at depth $z$ and $\sigma(z, t)$ is the shear stress among fluid layers.

As the thin plate move with prescribed speed $\dot{u}(t)=\dot{u}(0, t)$, the fluid exerts a shear stress $\sigma(0, t)=\sigma(t)$ on the thin plate that may be obtained in terms of the transverse velocity field of the viscous half space as in Fig. 1(a). In this regard, we analyze the transverse motion of a fluid column with area $A=1$ that is ruled by the solution of the parabolic differential equation for the transverse speed $\dot{u}(z, t)$

$$
\rho_{0} \ddot{u}(z, t)=\eta_{0} \frac{\partial^{2} \dot{u}(z, t)}{\partial z^{2}},
$$

where $\rho_{0}$ and $\eta_{0}$ are the mass density and the viscosity coefficient of the fluid, respectively. Equation (3.1) is supplemented by the initial condition $\dot{u}(z, 0)=0$, that is at $t=0$ the fluid is at rest. The boundary conditions of the model require that $u(\infty, t)=0$ (the influence of the plate's motion vanishes as $z \rightarrow \infty)$, and $\dot{u}(0, t)=\dot{u}(t)$, that is the fluid speed at $z=0$ equates the speed of the rigid plate. Once the transverse velocity field has been obtained the shear stress on the fluid is provided in the form

$$
\sigma(z, t)=\eta_{0} \frac{\partial \dot{u}(z, t)}{\partial z}
$$

By combining Eqs. (3.1) and (3.2) and using Laplace transform, after some algebra we get a relation among the shear force $F_{s}(t)$ for unitary area, namely, the shear stress, exerted by the fluid column on the rigid plate [Bagley and Torvik (1983); Schiessel and Blumen (1993)] given as

$$
F_{s}(t)=\sigma(t)=\sqrt{\rho_{0} \eta_{0}}\left({ }_{C} D_{0^{+}}^{1 / 2} \dot{u}\right)(t)=\sqrt{\rho_{0} \eta_{0}}\left({ }_{C} D_{0^{+}}^{3 / 2} u\right)(t) .
$$

That is the shear stress at the upper lamina exerted by the surrounding fluid is related to the speed of the lamina by a fractional derivative of order $1 / 2$. Such a model may be also considered, in the discretized version shown in Fig. 1(b), and it is represented by a sequence of rigid equispaced laminae of mass $\rho \Delta z$ connected by linear dashpots with dissipation coefficients $\eta / \Delta z$ and, as $\Delta z \rightarrow 0$, the equilibrium equation of each lamina, exactly, restitutes Eq. (3.1). The last equality in Eq. (3.3) shows that BT mechanical model does not yield a rheologic relation of viscoelasticity since the differentiation order $\beta \notin[0,1]$ and it may be considered as a visco-inertial (VI) fluid since its behavior is intermediate between pure fluid and pure inertial system.

A rheologic relation useful to describe VE solids may be obtained introducing slight changes in the BT model as shown in Fig. 2 reporting an unbounded elastic shear column 


\section{(a)}

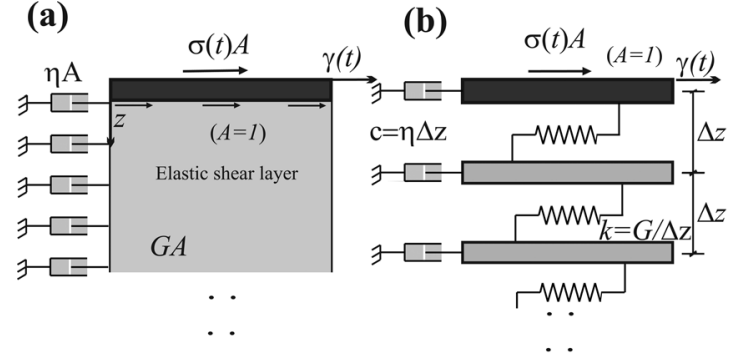

(c)

(d)

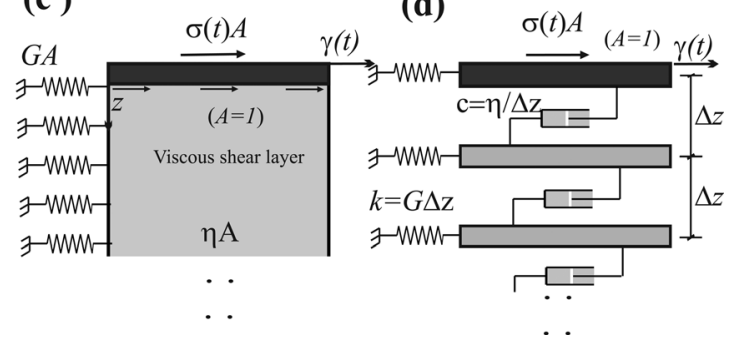

FIG. 2. (a) BT viscoelasticity; (b) discretized BT VE model; (c) BT elastoviscosity; and (d) discretized BT EV model.

of unitary cross-section $(A=1)$ with shear stiffness $G_{0} A=G_{0}\left(\left[G_{0}\right]=F L^{-2}\right)$ resting on a bed of linear dashpots with viscosity coefficient $\eta_{0} A\left(\left[\eta_{0}\right]=F T L^{-2}\right)$. A nondimensional coordinate system $z$ is introduced, $u(z, t)$ is considered in nondimensional form so that we may denote $u(0, t)=\gamma(t)$ and $u(z, t)=\gamma(z, t)$, and the external force field $F_{s}(t)=\sigma(t)$ $A=\sigma(t)$.

The equilibrium equation of the mechanical system shown in Fig. 2(a) in nondimensional coordinates and for unitary area reads

$$
\eta_{0} \dot{\gamma}(z, t)=G_{0} \frac{\partial^{2} \gamma(z, t)}{\partial z^{2}},
$$

yielding a relation among the shear stress $\sigma(t)$ and the shear strain $\gamma(0, t)=\gamma(t)$ at $z=0$ in the form

$$
\sigma(t)=G_{0}\left(\tau_{0}\right)^{1 / 2}\left({ }_{C} D_{0^{+}}^{1 / 2} \gamma\right)(t)
$$

with $\tau_{0}=\eta_{0} / G_{0}$ and it corresponds to a fractional rheologic model as we set $G_{0}\left(\tau_{0}\right)^{1 / 2}=C_{1 / 2}$ and $\beta=1 / 2$ in Eq. (2.8).

BT model may be obtained, also, as a limit case $(\Delta z \rightarrow 0)$ of the discrete system reported in Fig. 2(b) and composed by a sequence of massless plates interconnected by linear springs with stiffness $k_{0}=G_{0} A / \Delta z=G_{0} / \Delta z$ resting on a bed of viscous dashpots with viscosity $c_{0}=\eta_{0} A \Delta z=\eta_{0} \Delta z$. The only drawback beyond BT model is related to the fact that it restitutes, only, the particular value of the fractional derivative involved that is $\beta=1 / 2$.

The specular model of the modified BT mechanical description is represented by an indefinite massless viscous fluid, resting on a bed of independent springs reported in Fig. 2(c) and in discretized version in Fig. 2(d). As the viscous coefficient of the fluid and the stiffness of the springs are uniform, then, it may be easily proved that also in this case 


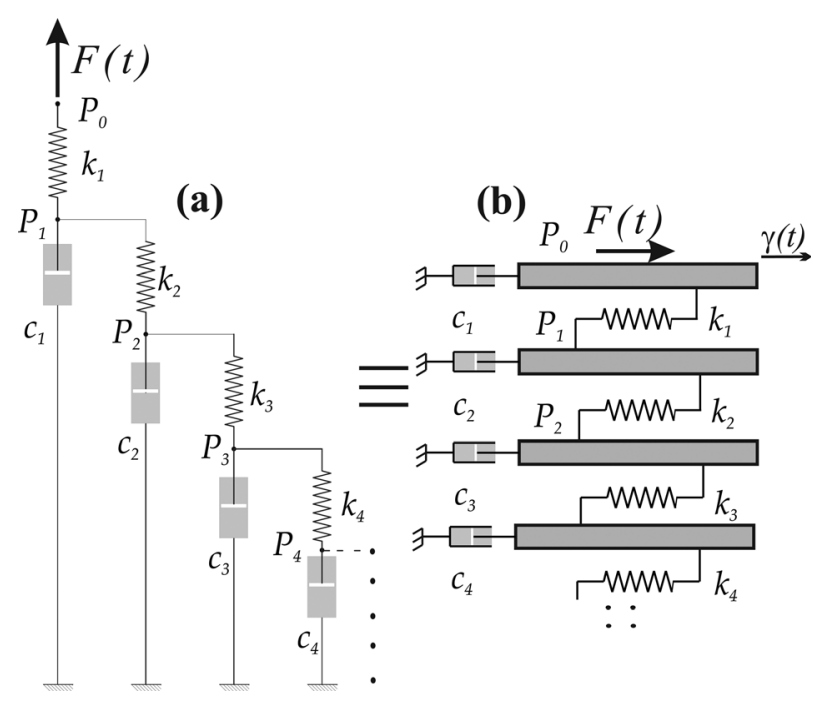

FIG. 3. (a) Original SB fractal ladder model; (b) equivalent shear-type mechanical system.

the shear stress $\sigma(t)$ exerted by the fluid on the upper lamina and the shear strain $\gamma(t)$ at the top of the fluid is related to the fractional derivative of order $\beta=1 / 2$. This is a crucial point that will be better discussed in Sec. V.

\section{B. The SB model}

The SB mechanical ladder is obtained assembling an infinite number of springs and Maxwell dashpots ordered hierarchically in a self-similar structure, a mechanical fractance that has been depicted in Fig. 3(a).

We denote the (normalized) displacements $\gamma_{0}(t), \gamma_{1}(t), \ldots \gamma_{k}(t), \ldots$ of the free nodes $P_{0}, P_{1}, \ldots P_{k}, \ldots$, respectively, and the displacement of the first element of the fractance [see Fig. 3(a)] is ruled by the differential equations system

$$
\left\{\begin{array}{l}
\sigma(t)=k_{1}\left(\gamma_{0}(t)-\gamma_{1}(t)\right) \\
k_{1}\left(\gamma_{0}(t)-\gamma_{1}(t)\right)=c_{1} \dot{\gamma}_{1}(t)+k_{2} \gamma_{1}(t)
\end{array}\right.
$$

yielding a relation, in Laplace domain, among the stress $\hat{\sigma}(s)$ and the correspondent normalized displacement $\hat{\gamma}(s)=\hat{\gamma}_{0}(s)$, that is

$$
\hat{\sigma}(s)=k_{1} \hat{\gamma}(s)\left(1-\frac{1}{f_{1}(s)}\right)
$$

where $f_{1}(s)=\tau_{1} s+r_{2}, \tau_{1}=c_{1} / k_{1}$ is a relaxation time and $r_{2}=k_{2} / k_{1}$ is a stiffness ratio. As we continue with the second element of the fractance in Fig. 3(b), we get a similar expression and iterating up to the j-element, the stress is related to the strain as

$$
\hat{\sigma}(s)=k_{1} \hat{\gamma}(s)\left(1-\frac{1}{f_{1}(s)-f_{2}(s)-f_{3}(s)-} \cdots \frac{r_{3}}{f_{j}(s)}\right),
$$

where $f_{j}(s)=\tau_{j} s+r_{j+1}, \tau_{j-1}=c_{j-1} / k_{j-1}$ and $r_{j}=k_{j} / k_{j-1}$ and the usual symbol denoting continues fractions has been used. The stress-strain relation of the fractal ladder discussed 
by $\mathrm{SB}$ is obtained as $j \rightarrow \infty$ yielding a constitutive law of the model in the form of an untermed continued fraction.

In SB fractal ladder, the scaling law of stiffness and damping coefficients of different elements of the fractance reads

$$
\begin{gathered}
k_{j}=\frac{1}{2 j-1} \frac{\Gamma(\beta)}{\Gamma(1-\beta)} \frac{\Gamma(j+1-\beta)}{\Gamma(j-1-\beta)} k_{1}, \\
c_{j}=2 \frac{\Gamma(\beta)}{\Gamma(1-\beta)} \frac{\Gamma(j+1-\beta)}{\Gamma(j+\beta)} c_{1} .
\end{gathered}
$$

By letting $j \rightarrow \infty$, the steady-state condition relating the imposed stress $\sigma(t)$ and the corresponding strain is obtained as

$$
\sigma(t) \cong \frac{k_{1}}{c_{1}^{\beta}}\left(D_{0^{+}}^{\beta} \gamma\right)(t)
$$

where $\left(D_{0^{+}}^{\beta} \gamma\right)(t)$ is the Riemann-Liouville fractional derivative.

The original SB model depicted in Fig. 3(a) seems, at first glance, to be quite different from the modified BT model depicted in Fig. 3(b). However, it may be observed that the two mechanical models are fully equivalent as we arrange springs and dashpots as in Fig. 3(b). It follows that the SB and the revisited BT model are equivalent in a mechanical perspective with the only difference that fractance in Fig. 3(b) introduces new springs and dashpots with prescribed scaling law [see, e.g., Eqs. (3.10) and (3.11)], while in the BT scheme in Fig. 3(b), all mechanical elements (springs and dashpots) of the model are identical.

Previous observations lead us to conclude that the exact mechanical model representing fractional hereditariness has to be composed by a half-space with proper arrangements of nonuniform springs and dashpots as it will be shown in Sec. IV.

\section{EXACT MODELS OF FRACTIONAL VISCOELASTICITY}

In this section, we introduce the exact mechanical models of fractional hereditariness considering that the interval of admissible values of $\beta$, namely, $\beta \epsilon[0,1]$ is composed by two disjoint intervals: $[0,1]=\left[0, \frac{1}{2}\right] \cup\left[\frac{1}{2}, 1\right]$. Differentiation order in ranges $0 \leq \beta \leq 1 / 2$ and $1 / 2 \leq \beta \leq 1$ produces different exact models dubbed, respectively, EV and VE materials. A wide discussion about the mechanical behavior beyond these different definitions will be discussed in Sec. V. We first examine VE materials $(1 / 2 \leq \beta \leq 1)$ because of the similarity with the modified BT model introduced in Sec. II.

\section{A. The fractional VE case $(1 / 2 \leq \beta \leq 1)$}

In this section, a mechanical model representing fractional rheologic stress-strain constitutive equation will be introduced in terms of the modified BT model with varying coefficients. Indeed, denoting $G_{V}(z) A=G_{V}(z)(A=1)$, the shear stiffness of the elastic half space and $\eta_{V}(z) A=\eta_{V}(z)$ the viscosity coefficients of the external dashpots as shown in Fig. 4. We assume that both $G_{V}(z)$ and $\eta_{V}(z)$ decay with power-law $z^{-\alpha}(0 \leq \alpha \leq 1)$ as

$$
G_{V}(z)=\frac{G_{0} z^{-\alpha}}{\Gamma(1-\alpha)} ; \quad 0 \leq \alpha \leq 1
$$


(a)

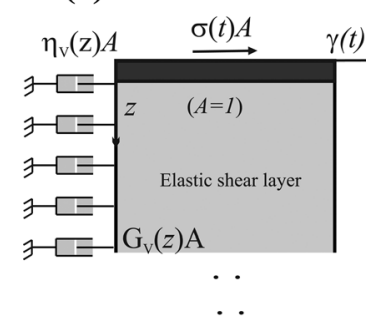

(b)

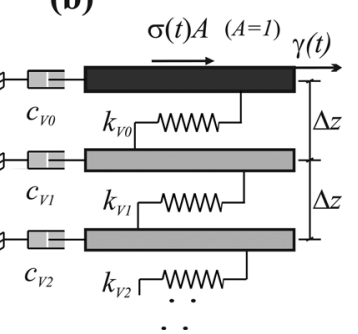

FIG. 4. (a) Continuous fractional VE model; (b) discretized representation of the fractional VE model.

$$
\eta_{V}(z)=\frac{\eta_{0}}{\Gamma(1+\alpha)} z^{-\alpha} ; \quad 0 \leq \alpha \leq 1
$$

The equilibrium equation at depth $z$ of the shear layer reads

$$
\frac{\partial}{\partial z}\left[G_{V}(z) \frac{\partial \gamma}{\partial z}\right]=\eta_{V}(z) \dot{\gamma}(z, t)
$$

that corresponds to discrete mechanical model composed by a shear-type frame interconnected by linear springs of stiffness $k_{V j}$ with external dashpots of stiffness $c_{V j}$ expressed as

$$
\begin{gathered}
k_{V j}=\frac{G_{0}}{\Gamma(1-\alpha)} \frac{z_{j}^{-\alpha}}{\Delta z}=\frac{G_{0}}{\Gamma(1-\alpha)} j^{-\alpha} \Delta z^{-\alpha-1} \\
c_{V j}=\frac{\eta_{0}}{\Gamma(1+\alpha)} z_{j}^{-\alpha} \Delta z=\frac{\eta_{0}}{\Gamma(1+\alpha)} j^{-\alpha} \Delta z^{1-\alpha} .
\end{gathered}
$$

The continuous VE model and its discretized counterpart are depicted in Fig. 5.

The equilibrium equation of the $j$ th shear layer of the discretized mechanical model is then provided in the form

$$
\left\{\begin{array}{l}
c_{V 0} \dot{\gamma}-k_{V 0} \Delta \gamma_{1}=\sigma(t) \\
c_{V j} \dot{\gamma}_{j}-k_{V j+1} \Delta \gamma_{j+1}+k_{V j} \Delta \gamma_{j}=0 ; \quad j=1,2, \ldots \infty
\end{array}\right.
$$

where $\gamma_{0}(t)=\gamma(t), \Delta \gamma_{j+1}=\gamma_{j+1}-\gamma_{j}$. By substituting Eqs. (4.4) and (4.5) into Eq. (4.6), at the limit, as $\Delta z \rightarrow 0$, Eq. (4.6) reverts, exactly, to Eq. (4.3). The continuous model

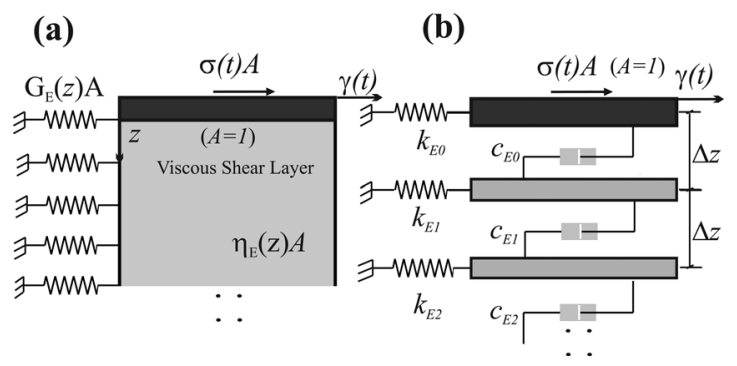

FIG. 5. (a) Continuous fractional EV model; (b) discretized representation of the fractional EV model. 
described in Eq. (4.3) yields a constitutive law in terms of Caputo's fractional derivative with order $1 / 2 \leq \beta \leq 1$. In order to show this, we perform Laplace transform of the normalized displacement field $\hat{\gamma}(z, s)=\mathcal{L}[\gamma]$ in Eq. (4.3) yielding an ordinary differential equation in Laplace domain in the form

$$
\frac{d}{d z}\left[k_{V}(z) \frac{d \hat{\gamma}(z, s)}{d z}\right]=s c_{V}(z) \hat{\gamma}(z, s)
$$

that may be solved, introducing Eqs. $(4.1,4.2)$ into Eq. (4.7) in terms of the first and the second modified Bessel functions (see Appendix B for details) denoted, respectively, $Y_{\beta}\left(z \sqrt{\tau_{V}(\alpha) s}\right)$ and $K_{\beta}\left(z \sqrt{\tau_{V}(\alpha) s}\right)$ as

$$
\hat{\gamma}(z, s)=z^{\beta}\left(B_{1} Y_{\beta}\left(z \sqrt{\tau_{\alpha}^{(V)} s}\right)+B_{2} K_{\beta}\left(z \sqrt{\tau_{\alpha}^{(V)} s}\right)\right),
$$

where we introduced the $\alpha$-dependent relaxation time $\tau_{\alpha}^{(V)}=-\eta_{0} \Gamma(-\alpha) /\left(\Gamma(\alpha) G_{0}\right)$ $=\tau_{0} \Gamma(-\alpha) / \Gamma(\alpha)$ and the index $\beta$ that reads

$$
\beta=\frac{(1+\alpha)}{2}
$$

Integration constants $B_{1}$ and $B_{2}$ in Eq. (4.8) are defined as we impose the relevant boundary conditions that reads

$$
\left\{\begin{array}{l}
\lim _{z \rightarrow 0} \hat{\gamma}(z, s)=\hat{\gamma}(s) \\
\lim _{z \rightarrow \infty} \hat{\gamma}(z, s)=0
\end{array}\right.
$$

yielding the integration constants

$$
B_{1}=0 ; \quad B_{2}=\frac{\hat{\gamma}(s)}{\Gamma(\beta) 2^{\beta-1}}\left(\tau_{\alpha}^{(V)} s\right)^{\beta / 2} .
$$

As the transverse displacement has been obtained, the shear stress at the top of the model is obtained with the relation (see Appendix B)

$$
\sigma(t)=C_{\beta}^{(V)}\left({ }_{C} D_{0^{+}}^{\beta} \gamma\right)(t)
$$

where we denoted

$$
G_{\beta}^{(V)}\left(\tau_{\alpha}^{(V)}\right)^{\beta}=C_{\beta}^{(V)}
$$

and the apparent shear modulus of the model reads

$$
G_{\beta}^{(V)}=\frac{G_{0} \Gamma(1-\beta)}{\Gamma(2(1-\beta)) \Gamma(\beta) 2^{1-2 \beta}} .
$$

It follows that the mechanical model of the modified BT representation with shear modulus and dashpot coefficients expressed as power-law corresponds, exactly, to a rheologic model in terms of Caputo' fractional derivatives. 
The mechanical model introduced in Fig. 5 corresponds, only, to values of the fractional differentiation order $\beta \in[1 / 2,1]$ since the decaying exponent of shear modulus and viscous coefficient $\alpha \epsilon[0,1]$ [see Eq. (4.9)]. Boundaries of exponent $\alpha$ have been introduced for two mechanical reasons: (i) values of $\alpha>1$ cannot be accepted since, in this case $\beta>1$ and the rheological model is no more related to a consistent VE material and (ii) negative values of the decay $\alpha$ cannot be accepted since, even though it may correspond to values of $\beta \in[0,1 / 2]$, the equilibrium at the top of the model cannot be satisfied since both $c_{V}(z)$ and $k_{V}(z)$ vanish at $z=0$. From these considerations, we conclude that the mechanical model for values of the derivative index $0 \leq \beta \leq 1 / 2$ has to be obtained with a different mechanical model. It is worth noticing that, limiting cases for $\alpha=0 \Rightarrow(\beta=1 / 2)$ and $\alpha=1 \Rightarrow(\beta=1)$, corresponds to the modified BT model presented in Sec. III A and to the case of a purely viscous fluid, respectively.

In passing we observe that the discrete counterpart of the mechanical model is analogous to the SB fractal ladder model with a different scaling law of springs and dashpots. That is the model proposed in this section is analogous to the fractal ladder model discussed in Schiessel and Blumen (1993) and Glockle and Nonnemacher (1991) with a different scaling law of springs and dashpots, and it represents a generalization of the BT model in Eq. (3.5).

\section{B. The fractional EV case $(0 \leq \beta \leq 1 / 2)$}

The arguments introduced in Sec. IV A led us to conclude that only differentiation orders $1 / 2 \leq \beta \leq 1$ may be covered with the pure shear elastic model resting on linear dashpots discussed in Sec. IV A. As a consequence differentiation order $0 \leq \beta \leq 1 / 2$ must be obtained with a different mechanical model with a different role among springs and dashpots. To this aim, we introduce an indefinite massless Newtonian fluid resting on a bed of independent springs (such as the Winkler model) depicted in Fig. 6.

The viscosity of the fluid $\eta_{E}(z)$ and the stiffness of the external springs $G_{E}(z)$, for unitary area, decay with power-law $z^{-\alpha}$ with $0 \leq \alpha \leq 1$ as

$$
\begin{aligned}
& G_{E}(z)=\frac{G_{0} z^{-\alpha}}{\Gamma(1+\alpha)} ; \quad 0 \leq \alpha \leq 1, \\
& \eta_{E}(z)=\frac{\eta_{0} z^{-\alpha}}{\Gamma(1-\alpha)} ; \quad 0 \leq \alpha \leq 1 .
\end{aligned}
$$

The equilibrium equation of the continuous model reads

$$
\frac{\partial}{\partial z}\left[\eta_{E}(z) \frac{\partial \dot{\gamma}}{\partial z}\right]=G_{E}(z) \gamma(z, t) .
$$

The discretized counterpart of such continuous model is composed by a shear-type frame interconnected by linear dashpots with external springs. The coefficient of viscosity of the dashpots between two adjacent floors located at depth $z_{j}=(j+1) \Delta z$ is provided in the form

$$
c_{E j}=\frac{\eta_{0}}{\Gamma(1-\alpha)} \frac{z_{j}^{-\alpha}}{\Delta z} ; \quad j=1,2, \ldots, \infty
$$

while the stiffness of the external springs located at the $j$ th shear layer, namely, $k_{E j}$ is defined as 

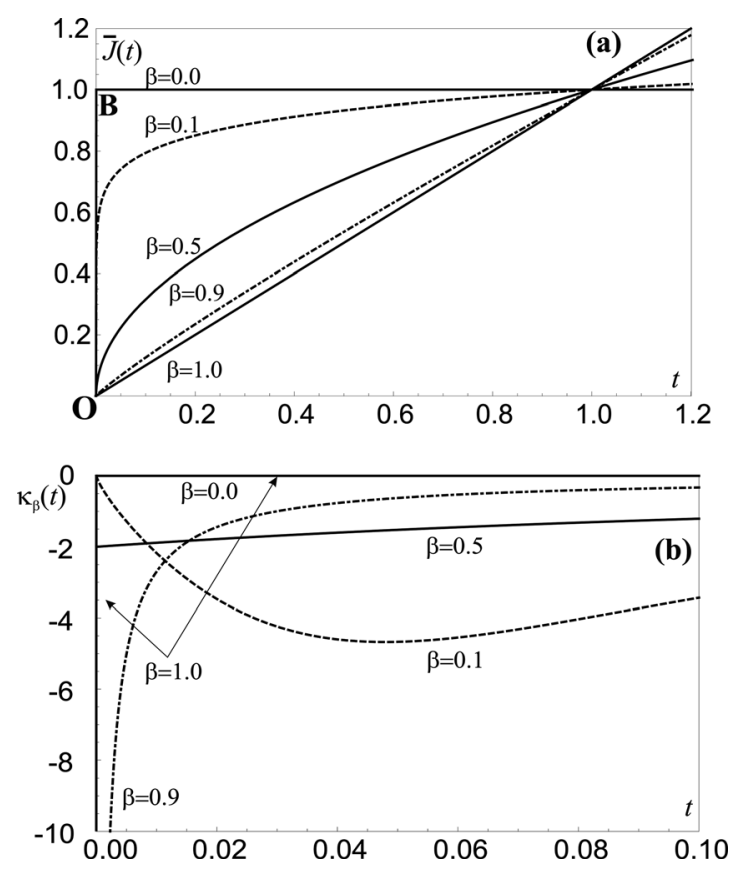

FIG. 6. (a) Normalized creep functions of fractional hereditary materials for different values of $\beta$; (b) curvatures of normalized creep functions for different values of the fractional derivative order $\beta$.

$$
k_{E j}=\frac{G_{0}}{\Gamma(1+\alpha)} z_{j}^{-\alpha} \Delta z ; \quad j=1,2, \ldots, \infty
$$

The equilibrium equation of the $j$ th shear layer may be written as

$$
\left\{\begin{array}{l}
k_{E 0} \gamma-c_{E 0} \Delta \dot{\gamma}_{1}=\sigma(t) \\
k_{E j} \gamma_{j}-c_{E j+1} \Delta \dot{\gamma}_{j+1}+c_{E j} \Delta \dot{\gamma}_{j}=0 ; \quad j=1,2, \ldots, \infty
\end{array}\right.
$$

where $\gamma_{0}=\gamma(t), \Delta \dot{\gamma}_{j+1}=\dot{\gamma}_{j+1}-\dot{\gamma}_{j}$. By inserting Eqs. (4.18) and (4.19) into Eq. (4.20), at the limit $\Delta z \rightarrow 0$ Eq. (4.20) reverts to Eq. (B3). The transverse displacement of the model is obtained in Laplace domain of Eq. (B3) yielding an ordinary differential equation

$$
\frac{d}{d z}\left[\eta_{E}(z) \frac{d \hat{\gamma}(z, t)}{d z}\right]=\frac{G_{E}(z) \hat{\gamma}(z, t)}{s}
$$

After some manipulations, a Bessel equation of second kind for the mapped field $\hat{\gamma}(z, s)=z^{\alpha} \bar{\gamma}(z, s)$ is obtained from Eq. (4.21) yielding the transverse displacement in terms of $\hat{\gamma}(z, s)$ as (see Appendix B for details)

$$
\hat{\gamma}(z, s)=z^{\beta}\left(B_{1} Y_{\beta}\left(\frac{z}{\sqrt{\tau_{\alpha}^{(E)} s}}\right)+B_{2} K_{\beta}\left(\frac{z}{\sqrt{\tau_{\alpha}^{(E)} s}}\right)\right),
$$

where $\tau_{\alpha}^{(E)}=-\tau_{0} \Gamma(\alpha) / \Gamma(-\alpha)$ is, dimensionally, a relaxation time. Evaluation of integration constants $B_{1}$ and $B_{2}$ in Eq. (4.22) is provided with the aid of boundary conditions in 
Eqs. (4.10) yielding the shear stress $\hat{\sigma}(s)$ at the top of the model in terms of the transverse displacement $\hat{\gamma}(s)$ as

$$
\hat{\sigma}(s)=G_{\bar{\beta}}^{(E)}\left(\tau_{\alpha}^{(E)}\right)^{\bar{\beta}} s^{\bar{\beta}} \hat{\gamma}(s)
$$

where $\bar{\beta}=1-\beta(0 \leq \bar{\beta} \leq 1 / 2)$ and the shear modulus $G_{\bar{\beta}}^{(E)}$ reads

$$
G_{\bar{\beta}}^{(E)}=\frac{G_{0} \Gamma(\bar{\beta})}{\Gamma(2-2 \bar{\beta}) \Gamma(1-\bar{\beta}) 2^{1-2 \bar{\beta}}} .
$$

The observation of Eq. (4.23) shows that the exponent of the Laplace parameter is a real number in $[0,1 / 2]$ yielding a rheologic stress-strain relation in time domain in the form

$$
\sigma(t)=C_{\bar{\beta}}^{(E)}\left({ }_{C} D_{0^{+}}^{\bar{\beta}} \gamma\right)(t)
$$

where $C_{\bar{\beta}}^{(E)}=G_{\bar{\beta}}^{(E)}\left(\tau_{E}(\alpha)\right)^{\bar{\beta}}$. Hence, we conclude that the mechanical model of EV material corresponds to the rheologic fractional model discussed in Eq. (2.7). Equation (4.25) allows to describe the purely elastic solid behavior assuming $\beta=1 \Rightarrow \bar{\beta}=0$ that is obtained as $\alpha=1$. In this context, the model corresponds to a sequence of shear layer externally restrained by linear springs of decreasing stiffness without internal connections.

At this stage, we may withdraw additional comments that represent fundamental consideration in the description of fractional VE rheologic models. The value $\beta=1 / 2$ of the derivation order separates two different ranges of material behavior: In the range $1 / 2 \leq \beta \leq 1$ in which the viscosity prevails, the elastic phase decreases with increasing $\beta$ and then it is appropriate to define such materials as VE. The corresponding mechanical model is composed by a shear indefinite column resting on a bed of linear dashpots. The second behavior is evidenced as $0 \leq \beta \leq 1 / 2$ in which the elastic phase prevails with decreasing $\beta$, and then it is appropriate to define these materials as EV ones. The corresponding mechanical model is described as an indefinite fluid resting on a bed of linearly independent springs. A detailed physical discussion about this point is reported in Sec. V.

The critical value of the fractional derivation order $\beta=1 / 2$ may be also obtained as a limit case $(\beta=1 / 2)$ for the two different models above described.

Additionally, we observe that the mechanical SB model is equivalent to the mechanical model reported in Sec. IV A and then the VE behavior may be captured in approximate form by those models. However, such a correspondence in the EV behavior is not equivalent to the mechanical model described in Sec. IV B. It follows that the EV behavior cannot be captured by the SB fractal ladder model and a different fractance-type structure must be considered by changing the role of springs and dashpots.

\section{A DEEPER INSIGHT IN FRACTIONAL HEREDITARY MATERIALS}

In Sec IV B, it has been shown that fractional hereditary materials (FHM) are represented by two distinct mechanical models that correspond to the same rheologic relation as $\beta=1 / 2$. In this section, the authors aim to investigate phenomenological and physical aspects behind the differences among EV and VE materials.

Let us consider the normalized creep function $\bar{J}(t)$ defined as

$$
\bar{J}(t)=J(t) C_{\beta} \Gamma(1+\beta)=t^{\beta},
$$

reported in Fig. 6(a) for different values of the exponent $\beta$. 
Normalized creep functions are monotonically increasing; As $t=0$ they have vertical slope for $0 \leq \beta \leq 1$ and all of them share a common point for $t=1$. However, since $\beta=1 / 2$ is a critical separation value among different mechanical behaviors this peculiarity must be reflected in the creep functions for $\beta<1 / 2$ and $\beta>1 / 2$. A first difference is that for EV materials and $0 \leq t \leq 1$ the creep function is always above the creep curve for $\beta=1 / 2$, and it remains below for $t>1$, whereas an opposite trend is evidenced for VE materials. A physical picture of this behavior may be provided as we observe that the value of time instant $t=1$ corresponds to a crossover temporal value of EV and VE materials with respect to the critical value $(\beta=1 / 2)$. Indeed, EV materials show a rheological behavior intermediate among a pure solid $(\beta=0)$ and FHM with critical order $\beta=1 / 2$. In this case, it may be observed that at the beginning $(0 \leq t \leq 1)$, the normalized transverse displacement follows the applied constant actions (similar to an elastic solid), and the creep curve is over the case $\beta=1 / 2$. As $t \geq 1$, the viscous phase becomes predominant so that increments of material displacement decelerates and the creep function is below the critical case $\beta=1 / 2$. VE materials show an opposite trend since the rheological features of the material are intermediate among a pure fluid $(\beta=1)$ and the critical value $(\beta=1 / 2)$. Indeed, as $0 \leq t \leq 1$, the materials behave like a pure fluid showing a progressive increment of the transverse displacement with time, due to the viscous phase and the creep function is below the curve with $(\beta=1 / 2)$. As $t \geq 1$, instead, the presence of the elastic phase tends to accelerate the increment of the transverse displacement with respect to the critical material hereditariness $(\beta=1 / 2)$, and the creep curve is over the case $(\beta=1 / 2)$.

More evident geometrical differences among EV and VE materials may be observed on the curvature $\kappa_{\beta}(t)$ of $\bar{J}(t)$ provided as

$$
\kappa_{\beta}(t)=\frac{\beta(\beta-1) t^{\beta-2}}{\left(1+\beta^{2} t^{2(\beta-1)}\right)^{3 / 2}}
$$

Curvature $\kappa_{\beta}(t)$ has been depicted in Fig. 6(b) for different values of $\beta$ and very small $t$. It must be observed that, for $0 \leq \beta \leq 1 / 2$, all curvatures $\lim _{t \rightarrow 0}\left[\kappa_{\beta}(t)\right]=0$ decrease up to a minimum value at time instant $t_{\beta}^{\star}$ and then monotonically increase up to zero as $t \rightarrow \infty$. If $\beta=1 / 2$, then $\lim _{t \rightarrow 0}\left[\kappa_{\beta}(t)\right]=-2$ and then monotonically increase as $\lim _{t \rightarrow \infty} \kappa_{1 / 2}(t)=0$.

A different situation may be observed for VE materials since $\lim _{t \rightarrow 0}\left[\kappa_{\beta}(t)\right]=-\infty$ and monotonically increase reaching zero as $t \rightarrow \infty$. With these considerations in mind we preliminary observe that the purely elastic behavior start in zero (point 0 in Fig. 6(a)) with unbounded slope, at the point B in Fig. 6(a), the slope drops down to zero and the curvature remains zero as $t>0$, while the curvature attains a minimum in $\mathrm{B}$ that is $-\infty$.

The EV materials start in zero with unbounded slope (as the pure solid), the curvature in zero is zero (like the pure solid at point $\mathrm{O}$ ), then as the time instant reaches $t_{\beta}^{\star}$ the curve remains monotonic. The point corresponding to the maximum curvature corresponds to point B of the purely elastic solid. From these considerations, we may affirm that, for very small values of $t$ the pure solid behavior prevail in the EV materials. As $t>t_{\beta}^{\star}$, the viscous trend influences more and more the EV behavior.

A different scenario appears for VE materials as it may be observed from Fig. 6(b). In fact, the curvature in zero as $1 / 2 \leq \beta \leq 1$ is $-\infty$ and then the curvature monotonically tends to zero as $t \rightarrow \infty$. This behavior is quite similar to the pure viscous fluid for which, at $t \rightarrow 0$, the curvature is $-\infty$ (singular point), and then it remains zero for $t>0$. Indeed, as $t=0$, the slope for $\beta>1 / 2$ is unbounded, because the curvature is $-\infty$, for very small 
values of $t$ we may affirm that the VE material behaves like a pure fluid. Then, we conclude that for $\beta>1 / 2$ the viscous behavior prevails.

For $\beta=1 / 2$, the minimum curvature is attained as $t=0$, where the value of curvature is -2 , and then the curvature monotonically increases and this is an intermediate behavior between VE and EV materials since $\lim _{t \rightarrow 0}\left[\kappa_{\beta}(t)\right]$ is neither 0 nor $-\infty$ but it is a finite value.

All these considerations have been made simply by direct inspection of the creep curve and bearing in mind that $\beta=1 / 2$ is a critical value; some other insights about the mechanical behavior of the EV and VE materials may now be withdrawn.

EV material model possesses a mechanical representation in terms of fluid layers restrained by external springs. The springs and dashpots decay with power-law as the depth increases, then the model is free and the global equilibrium is guaranteed only by the external springs. It follows that, when the top layer experiences a load history $\sigma(t)=U(t)$ (creep test) then in proximity of $t=0$, the fluid behaves like a rigid solid and then all the external springs restitute a load proportional to their own stiffness. In this phase, the EV material behaves like a solid in the point O shown in Fig. 6(a). As soon as the time increases the dashpots between adjacent layers start to experience a velocity gradient among the layers and the motion begins giving in time the constitutive law described by the creep function.

The VE material model is a free unbounded elastic shear column solid resting on a bed of viscous dashpots. At time $t=0$, the unforced stress at the top of the model is constrained only by the external dashpots since both the displacements and the relative displacements are very small, and then the internal mutual interactions are nearly vanishing and this justifies the unbounded slope in zero. As $t$ increases, each dashpots move with different velocities then the internal springs start to redistribute the internal forces. This effect is highlighted as the relative displacement among adjacent layers increases as it happens for large values of $t$. It follows that, in the first few instants, the viscous fluid behavior prevails over the elastic one and this justify the definition of VE materials for $\beta>1 / 2$.

As a conclusion, the two mechanical models behave in a very different fashion especially for small values of $t$ during the creep test. The critical point for $\beta=1 / 2$ may be obtained as a limit $\beta \rightarrow 1 / 2$ from the left (EV) material or from the right (VE) material, that is the behavior trend passes from EV to VE with continuity.

Similar considerations may be also withdrawn from the relaxation curves, but they have not been reported for shortness sake's.

\section{CLOSURE AND DISCUSSION}

In this paper, two exact mechanical models describing fractional hereditary materials have been presented. The two models cover the entire range of the derivation order $\beta \varepsilon[0,1]$. FHM with $0 \leq \beta \leq 1 / 2$ have been dubbed $\mathrm{EV}$ and the equivalent mechanical model is composed by an unbounded viscous fluid column resting on a bed of linear springs. FHM with $1 / 2 \leq \beta \leq 1$ have been dubbed VE and they possess a mechanical model represented by an unbounded shear-type column resting on a bed of linear dashpots. The constitutive law obtained introducing at the top of the model an assigned load history and measuring the corresponding strain, restitutes, exactly, the well-known fractional constitutive law.

In virtue of such a consideration several comments may be withdrawn:

(1) The $\mathrm{BT}$ model in its original form gives an exact mechanical description for $\beta=3 / 2$. Here, with convenient modifications, the derivation order has been reduced to yield a 
model that provides a derivation order $\beta=1 / 2$ so reproducing a true VE behavior. This modified version coalesces with the exact model presented in the paper for $\beta=1 / 2$.

(2) The discretized version of the BT model, from a mechanical perspective, is equivalent to the fractal ladder model proposed by SB. That is both are obtained as massless, shear-type, indefinite frames resting on a bed of independent dashpots. The only real difference is related with the different laws of variation of stiffness and viscosity from adjacent shear layers.

(3) The exact mechanical model here proposed in discretized form is quite similar to the SB model for values of $\beta$ in the interval $1 / 2 \leq \beta \leq 1$. It is represented by an undefined massless shear frame resting on a bed of independent dashpots with a powerlaw decay of stiffness and viscosity coefficient as the distance between the top of the frame increase. Values of $\beta$ in the interval $0 \leq \beta \leq 1 / 2$, the exact mechanical model is the same as in case $1 / 2 \leq \beta \leq 1$ with an opposite role of springs and dashpots.

(4) During the few time instants of creep test, the mechanical models behave in a different way: As $0 \leq \beta \leq 1 / 2$, the material behaves as a solid whereas for $1 / 2 \leq \beta \leq 1$ the fractional-type material behaves like a fluid. The different mechanical behaviors suggest to define EV materials for $0 \leq \beta \leq 1 / 2$ and VE materials for $1 / 2 \leq \beta \leq 1$.

(5) Differentiation order $\beta=1 / 2$ corresponds to critical-order hereditariness and the mechanical model may be obtained at the limit as $\beta$ increases from the left (EV) or $\beta$ decreases from the right (VE). That is the transition among the two regimes is continuous with $\beta$.

(6) The results provided in the paper open new perspectives on fractional operators. Such an example, in presence of differentiation order, $1 \leq \beta \leq 2$, the mechanical model is represented by an indefinite fluid with density and viscosity coefficients that decay with power-law. But also in this case the value $\beta=3 / 2$ separates two different cases that now, we recognize as VI and inertial-viscous (IV) fluids. Analogous considerations may be exported in the field of nonlocal continuum mechanics as well as in nonlocal thermal energy transport where nonlocal contributions are modeled as power-laws of the interdistances among interacting locations.

The discretized versions of the continuous models have also been analyzed, and several numerical analyses have been performed by contrasting the results with GrnwaldLetnikov representation of fractional integration scheme for fractional constitutive laws. Such results have been not reported for shortness' sake. But the beauty of such results allow us to affirm that the discretized versions of those two continuous models are capable to provide a physical interpretation of fractional operators as well as they may be used for practical calculations. This issue will be addressed in a future paper.

\section{ACKNOWLEDGMENTS}

The authors are very grateful to research Grant No. PRIN2008, National Coordinator Professor A. Carpinteri. This financial support is gratefully acknowledged.

\section{APPENDIX A: FRACTIONAL OPERATORS}

In this appendix, we report a brief outline of the main steps used to derive Eq. (4.8) as well as fundamental definitions used in the paper about real-order (fractional) operators. The Euler-Gamma function $\Gamma(\beta)$ may be considered as the generalization of the factorial function since, as $\beta$ assumes integer values as $\Gamma(n+1)=n$ ! and it is defined as the result of the integral 


$$
\Gamma(\beta)=\int_{0}^{\infty} \exp (-z) z^{\beta-1} d z
$$

Riemann-Liouville fractional integrals and derivatives defined on the entire real axis $\mathbb{R}$ are defined as

$$
\begin{aligned}
& \left(I_{+}^{\beta} f\right)(t)=\frac{1}{\Gamma(\beta)} \int_{-\infty}^{t} \frac{f(\tau)}{(t-\tau)^{1-\beta}} d \xi \\
& \left(D_{+}^{\beta} f\right)(t)=\frac{d}{d t}\left[\left(I_{+}^{1-\beta} f\right)(t)\right]=\frac{1}{\Gamma(1-\beta)} \int_{-\infty}^{t} \frac{f(\tau)^{\prime}}{(t-\tau)^{\beta}} d \xi
\end{aligned} .
$$

Riemann-Liouville fractional integrals and derivatives of functions defined over intervals of the real axis, namely, $f(t) \epsilon[a, b] \subset \mathbb{R}$ reads

$$
\begin{aligned}
& \left(I_{a^{+}}^{\beta} f\right)(t)=\frac{1}{\Gamma(\beta)} \int_{a}^{t} \frac{f(\tau)}{(t-\tau)^{1-\beta}} d \xi \\
& \left(D_{a^{+}}^{\beta} f\right)(t)=\frac{d}{d t}\left[\left(I_{a^{+}}^{1-\beta} f\right)(t)\right]=\frac{f(a)}{\Gamma(1-\beta)(t-a)^{\beta}}+\frac{1}{\Gamma(1-\beta)} \int_{a}^{t} \frac{f(\tau)^{\prime}}{(t-\tau)^{\beta}} d \tau
\end{aligned}
$$

Beside Riemann-Liouville fractional operators defined in Eqs. (A2) and (B2) another class of integrodifferential operators that are often used in the context of fractional viscoelasticity is represented Caputo fractional derivatives $\left({ }_{C} D_{a^{+}}^{\beta} f\right)(t)$ that are defined as

$$
\left({ }_{C} D_{a^{+}}^{\beta} f\right)(t)=\frac{1}{\Gamma(1-\beta)} \int_{a}^{t} \frac{f(\tau)^{\prime}}{(t-\tau)^{\beta}} d \tau
$$

that coincides with the integral part of the Riemann-Liouville fractional derivative reported in Eq. (A4) in unbounded domain.

The observation of Eqs. (B2) and (A4) shows that under mild conditions for the function class $f(t)$, and under the assumption $f(a)=0$, Caputo and Riemann-Liouville fractional derivatives coalesce. Similar considerations hold true also for Caputo and Riemann-Liouville fractional derivatives defined on the entire real axis. As a consequence, Caputo fractional integral is perfectly coalescing, in bounded and unbounded domains, with Riemann-Liouville fractional integral and it is not defined in scientific literature. Caputo fractional derivatives may be consider as the interpolation among the well-known, integer-order derivatives, operating over functions $f(t)$ that belong to the class of Lebesgue integrable functions $L^{(1)}$, that is, $f(t) \in L^{(1)}$ and, as a consequence, they are very useful in the mathematical description of complex systems' evolution.

The Laplace $\mathcal{L}[\bullet]$ and Fourier $\mathcal{F}[\bullet]$ integral transforms of Caputo fractional derivatives read, respectively,

$$
\left\{\begin{array}{l}
\mathcal{L}\left[\left({ }_{c} D_{0^{+}}^{\beta} f\right)(t)\right]=s^{\beta} \mathcal{L}[f(t)]=s^{\beta} \hat{f}(s) \\
\mathcal{F}\left[\left({ }_{c} D_{+^{+}}^{\beta} f\right)(t)\right]=(-i \omega)^{\beta} \mathcal{F}[f(t)]=(-i \omega)^{\beta} \tilde{f}(\omega)
\end{array},\right.
$$

where the Laplace and Fourier transforms are defined as 


$$
\left\{\begin{array}{c}
\mathcal{L}[f(t)]=\hat{f}(s)=\int_{0}^{\infty} f(t) \exp (-t s) d t \\
\mathcal{F}[f(t)]=\tilde{f}(\omega)=\int_{-\infty}^{\infty} f(t) \exp (-i \omega t) d t
\end{array} .\right.
$$

The Laplace and Fourier transforms of Riemann-Liouville fractional integrals reads

$$
\left\{\begin{array}{l}
\mathcal{L}\left[\left(I_{0^{+}}^{\beta} f\right)(t)\right]=s^{-\beta} \mathcal{L}[f(t)]=s^{-\beta} \hat{f}(s) \\
\mathcal{F}\left[\left(I_{+^{+}}^{\beta} f\right)(t)\right]=(-i \omega)^{-\beta} \mathcal{F}[f(t)]=(-i \omega)^{-\beta} \tilde{f}(\omega)
\end{array} .\right.
$$

\section{APPENDIX B: BESSEL DIFFERENTIAL EQUATIONS}

Ordinary differential equation reported in Eq. (4.8) may be solved as we perform differentiations at the left hand side yielding

$$
\frac{d^{2} \hat{\gamma}(s, z)}{d z^{2}}+\frac{k_{V}^{\prime}(z)}{k_{V}(z)} \frac{d \hat{\gamma}(s, z)}{d z}-\frac{c_{V}(z)}{k_{V}(z)} s \hat{\gamma}(s, z)=0,
$$

and replacing for the stiffness coefficient $k_{V}(z)$ and viscous coefficient $c_{V}(z)$ the corresponding power-laws reported in Eqs. (4.1) and (4.2) the differential equation ruling the unitary transverse displacement of the system reads

$$
\frac{d^{2} \hat{\gamma}(s, z)}{d z^{2}}-\frac{\alpha}{z} \frac{d \hat{\gamma}(s, z)}{d z}-\tau_{0} s \hat{\gamma}(s, z)=0
$$

The governing equation of the transverse displacement may be reverted into a Bessel equation of the second kind introducing an auxiliary function $\bar{\gamma}(z, s)$ related to the unknown function $\hat{\gamma}(z, s)$ by means of the nonlinear mapping $\hat{\gamma}(z, s)=z^{\alpha} \bar{\gamma}(z, s)$ so that the first and second-order derivatives involved in Eq. (B2) read

$$
\begin{gathered}
\frac{d \hat{\gamma}(z, s)}{d z}=\alpha z^{\alpha-1} \bar{\gamma}(z, s)+z^{\alpha} \frac{d \bar{\gamma}(z, s)}{d z}, \\
\frac{d^{2} \hat{\gamma}(z, s)}{d z^{2}}=\frac{d}{d z}\left[\alpha z^{\alpha-1} \bar{\gamma}(z, s)+z^{\alpha} \frac{d \bar{\gamma}(z, s)}{d z}\right] \\
=\alpha(\alpha-1) z^{\alpha-2} \bar{\gamma}(z, s)+2 \alpha z^{\alpha-1} \frac{d \bar{\gamma}(z, s)}{d z}+z^{\alpha} \frac{d^{2} \bar{\gamma}(z, s)}{d z^{2}},
\end{gathered}
$$

and substitutions into Eq. (B2) yield a modified Bessel equation for function $\bar{\gamma}(z, s)$ as

$$
z^{2} \frac{d^{2} \bar{\gamma}(z, s)}{d z^{2}}+\alpha z \frac{d \bar{\gamma}(z, s)}{d z}-\left(z^{2} \tau_{\alpha}^{(V)} s+\alpha\right) \bar{\gamma}(z, s)=0
$$

where $\tau_{\alpha}^{(V)}=-\tau_{0} \frac{\Gamma(-\alpha)}{\Gamma(\alpha)}$. Second-order differential equation for the function $\bar{\gamma}(z, s)$ in Eq. (B5) is solved in terms of the modified first and second Bessel functions, namely, $Y_{\beta}(z)$ and $K_{\beta}(z)$ yielding 


$$
\bar{\gamma}(z, s)=z^{\frac{1-\alpha}{2}}\left(B_{1} Y_{\beta}\left(z \sqrt{\tau_{\alpha}^{(V)} s}\right)+B_{2} K_{\beta}\left(z \sqrt{\tau_{\alpha}^{(V)} s}\right)\right)
$$

where Bessel functions are defined as extension of power-series expansion of classical exponentials as

$$
Y_{\beta}(z)=\sum_{k=0}^{\infty} \frac{(z / 2)^{\beta+2 k}}{k ! \Gamma(k+\beta+1)} \quad K_{\beta}(z)=\frac{\pi}{2 \sin (2 \pi \beta)}\left[Y_{-\beta}(z)-Y_{\beta}(z)\right],
$$

and with $B_{1}, B_{2}$ unknown integration constants that depend on the relative boundary conditions in Eq. (4.10).

The strain field $\hat{\gamma}(z, s)$ is then expressed by the relation

$$
\hat{\gamma}(z, s)=\frac{\hat{\gamma}(s)}{\Gamma(\beta) 2^{\beta-1}}\left(\tau_{\alpha}^{(V)} s\right)^{\beta / 2} z^{\beta} K_{\beta}\left(z \sqrt{\tau_{\alpha}^{(V)} s}\right)
$$

that corresponds to a shear stress among adjacent locations of the elastic shear layer in the form

$$
\hat{\sigma}(z, s)=k_{V}(z) \frac{\partial \hat{\gamma}(z, s)}{\partial z}=G_{\beta}^{(V)} \hat{\gamma}(s)\left(\tau_{\alpha}^{(V)} s\right)^{\frac{1+\beta}{2}} z^{1-\beta} K_{\beta-1}\left(z \sqrt{\tau_{\alpha}^{(V)} s}\right)
$$

where the $\beta$-dependent apparent elastic shear modulus of the VE model, namely, $G_{\beta}^{(V)}$ is reported in Eq. (4.14). The stress-strain relationship at $z=0$ is then provided at the limit $\hat{\sigma}(0, s)=\hat{\sigma}(s)=\lim _{z \rightarrow 0} \hat{\sigma}(z, s)$ as

$$
\hat{\sigma}(s)=G_{\beta}^{(V)}\left(\tau_{\alpha}^{(V)} s\right)^{\beta} \hat{\gamma}(s)
$$

that in the time domain restitutes the fractional rheologic model of VE material in Eq. (4.12).

A similar approach may be used to obtain the fractional rheologic model of EV material. In this case, differentiation of Eq. (4.21) yields

$$
\frac{d^{2} \hat{\gamma}(s, z)}{d z^{2}}+\frac{\eta_{E}^{\prime}(z)}{\eta_{E}(z)} \frac{d \hat{\gamma}(s, z)}{d z}-\frac{G_{E}(z)}{s \eta_{E}(z)} \hat{\gamma}(s, z)=0,
$$

and replacing the decay of stiffness and viscosity along the fluid column reported in Eqs. (4.15) and (4.16) into Eq. (B11), it yields

$$
\frac{d^{2} \hat{\gamma}(s, z)}{d z^{2}}-\frac{\alpha}{z} \frac{d \hat{\gamma}(s, z)}{d z}-\frac{1}{s \tau_{\alpha}^{(E)}} \hat{\gamma}(s, z)=0
$$

where $\tau_{\alpha}^{(E)}=-\tau_{0} \frac{\Gamma(\alpha)}{\Gamma(-\alpha)}$. As we introduce the nonlinear mapping $\hat{\gamma}(z, s)=z^{\alpha} \bar{\gamma}(z, s)$ with the aid of Eqs. (B3) and (B4) a Bessel differential equation of second kind is obtained as

$$
z^{2} \frac{d^{2} \bar{\gamma}(z, s)}{d z^{2}}+\alpha z \frac{d \bar{\gamma}(z, s)}{d z}-\left(\frac{z^{2}}{\tau_{\alpha}^{(E)} s}+\alpha\right) \bar{\gamma}(z, s)=0 .
$$


Solution of Eq. (B13) is obtained in terms of the modified first and second Bessel functions $Y_{\beta}(z)$ and $K_{\beta}(z)$ as

$$
\bar{\gamma}(z, s)=z^{\frac{1-\alpha}{2}}\left(B_{1} Y_{\beta}\left(\frac{z}{\sqrt{\tau_{\alpha}^{(E)} s}}\right)+B_{2} K_{\beta}\left(\frac{z}{\sqrt{\tau_{\alpha}^{(E)} s}}\right)\right),
$$

with boundary conditions coalescing with Eq. (4.10). As we require fulfillment of the boundary conditions we get values of the integration constant in Eq. (4.22) leading to $B_{1}=0$ and

$$
B_{2}=\frac{\hat{\gamma}(s) 2^{1-\beta}}{\Gamma(\beta)}\left(\tau_{\alpha}^{(E)} s\right)^{-\beta / 2}
$$

so that the transverse displacement field of the model is expressed as

$$
\hat{\gamma}(z, s)=\frac{2^{1-\beta} \hat{\gamma}(s)}{\Gamma(\beta)}\left(\tau_{\alpha}^{(E)} s\right)^{-\beta / 2} z^{\beta} K_{\beta}\left(\frac{z}{\sqrt{\tau_{\alpha}^{(E)} s}}\right) .
$$

The Laplace transform of the stress field at a prescribed depth $z$ is given, after some algebraic manipulations, in the form

$$
\hat{\sigma}(z, s)=c_{E}(z) s \frac{\partial \hat{\gamma}(z, s)}{\partial z}=G_{\beta}^{(E)}\left(\tau_{\alpha}^{(E)} s\right)^{\frac{1-\beta}{2}} z^{1-\beta} K_{\beta-1}\left(\frac{z}{\sqrt{\tau_{\alpha}^{(E)} s}}\right),
$$

where $G_{\beta}^{(E)}$ has been reported in Eq. (4.24).

The stress field at $z=0$ is obtained as $\hat{\sigma}(0, s)=\hat{\sigma}(s)=\lim _{z \rightarrow 0} \hat{\sigma}(z, s)$ yielding

$$
\hat{\sigma}(s)=G_{\bar{\beta}}^{(E)}\left(\tau_{\alpha}^{(E)} s\right)^{\bar{\beta}} \hat{\gamma}(s)
$$

that corresponds to the fractional rheologic model expressed in Eq. (4.25).

\section{References}

Bagley, R. L., and P. J. Torvik, "Fractional calculus-A different approach to analysis of viscoelastically damped structures," AIAA J. 21, 741-748 (1983).

Bagley, R. L., and P. J. Torvik, "On the fractional calculus model of viscoelastic behavior," J. Rheol. 30, 133-155 (1986).

Blizard, R. B., "Visco-elasticity of rubber," J. Appl. Phys. 22, 730-735 (1951).

Caputo, M. G., "Vibrations of a thin viscoelastic layer with a dissipative memory," J. Acoust. Soc. Am. 56, 897-904 (1974).

Friedrich, C., "Relaxation and retardation functions of the Maxwell model with fractional derivatives," Rheol. Acta 30, 151-158 (1991).

Friedrich, C., "Mechanical stress relaxation in polymers: Fractional integral model versus fractional differential model," J. Non-Newtonian Fluid Mech. 46, 307-314 (1993).

Friedrich, C., and H. Braun, "Linear viscoelastic behaviour of complex polymeric materials: A fractional mode representation," Colloid Polym. Sci. 272, 1536-1546 (1994). 
Glockle, W. G., and T. F. Nonnenmacher, "Fractional integral operators and fox functions in the theory of viscoelasticity," Macromolecules 24, 6426-6434 (1991).

Heymans, N., "Constitutive equations for polymer viscoelasticity derived from hierarchical models in cases of failure of time-temperature superposition," Signal Process. 83, 2345-2357 (2003).

Heymans, N., and J. C. Bauwens, "Fractal rheological models and fractional differential equations for viscoelastic behavior," Rheol. Acta 33, 210-219 (1994).

Mainardi, F., Fractional Calculus and Waves in Linear Viscoelasticity (Imperial College, London, 2010).

Metzler, H. R., A. Blumen, and T. F. Nonnenmacher, "Generalized viscoelastic models: Their fractional equations with solutions," J. Phys. A 28, 6567-6584 (1995a).

Metzler, H. R., and J. Klafter, "The random walk guide to anomalous diffusion: A fractional dynamics approach,” Phys. Rep. 339, 1-77 (2000).

Metzler, H. R., and T. F. Nonnenmacher, "Fractional relaxation processes and fractional rheological models for the description of a class of viscoelastic materials," Int. J. Plast. 19, 941-959 (2003).

Metzler, H. R., W. Schick, H. G. Kilian, and T. F. Nonnenmacher, "Relaxation in filled polymers: A fractional calculus approach,” J. Chem. Phys. 103, 7180-7186 (1995b).

Nutting, P. G., “A new general law deformation,” J. Franklin Inst. 191, 678-685 (1921).

Podlubny, I., Fractional Differential Equations (Academic, New York, 1998).

Rouse, P. E., and K. Sittel, "Viscoelastic properties of dilute polymer solutions," J. App. Phys. 24, 690-696 (1953).

Sakakibara, S., "Relaxation properties of fractional derivative viscoelastic models," Nonlinear Anal. 47, 5449-5454 (2001).

Samko, G. S., A. A. Kilbas, and O. I. Marichev, Fractional Integrals and Derivatives (Gordon and Breach Science, Amsterdam, 1993).

Scott-Blair, G., "An application of the theory of quasi-properties to the treatment of anomalous stress-strain relation," Phys. Mag. 40, 80-94 (1949).

Schiessel, H., and A. Blumen, "Hierarchical analogues to fractional relaxation equations," J. Phys. A 26, 5057-5069 (1993).

Schiessel, H., and A. Blumen, "Mesoscopic pictures of the sol-gel transition: Ladder models and fractal networks," Macromolecules 28, 4013-4019 (1994).

Spanos, P. D., and G. I. Evangelatos, "Response of a non-linear system with restoring forces governed by fractional derivatives-time domain simulation and statistical linearization solution," Soil. Dyn. Earthquake Eng. 30, 6426-6434 (1991). 